\title{
Control systems for unmanned combine harvester
}

Zahid Godzhaev ${ }^{1}$, Y.P. Lobachevsky ${ }^{1}$, Ilya Alekseev ${ }^{1}$, Alexander Prilukov ${ }^{1}$, and T.Z. Godzhaev $^{1}$

${ }^{1}$ FSBSI Federal Scientific Agroengineering Center VIM, 1st Institutsky proezd, 5, Moscow, 109428, Russia

\begin{abstract}
Nowadays, there are many technical and technological solutions to increase productivity in agriculture. One of such solutions is the use of the latest robotic mobile systems that can increase agricultural productivity. For the implementation of these decisions, sound concepts are needed to create control systems for agricultural unmanned mobile energy devices based on an analysis of current trends in the development of robotic mobile energy devices. This paper discusses various areas of the concept of creating unmanned control systems for a mobile energy device a combine harvester, ensuring high-quality performance of technological operations of harvesting, including the directional stability of the combine harvester. The most effective version of the concept of creating a control system was selected.
\end{abstract}

\section{Introduction}

In recent years, in connection with the rapid development of automation and robotization of various machine-technological processes, the adjective "intelligent" and, accordingly, intelligent control systems have been actively used. One of the main examples of the implementation of these systems is robotics, in particular, unmanned driving of mobile energy and transport-technological devices.

An analysis of the current state and development trends of mobile technical devices, for brevity we will use the term mobile robotic complex (MRC), used in various fields of human life, shows that most of the technologies used to create robotic complexes are based on configuration space [1].

The aim of the study is to develop a concept for creating an effective management system (CS) for unmanned MRC based on an analysis of current trends in the development of robotic mobile transport and technological devices that provide the necessary operational and functional properties of MRC in agricultural production.

\section{Concept analyses}

For a constructive discussion of the concepts, methods and technologies for developing effective MRC control systems for agricultural purposes, it is proposed to reduce the dimension of the initial technology space and consider the assembly point of robots with a 
high degree of autonomy in the space of MRC configurations. The research results are applicable to all of the listed categories of MRC. In the concept being developed, in addition to the accurate execution of technological operations with the help of the developed control system for unmanned driving, safety issues should be worked out during the execution of technological operations. An enlarged block diagram of the control object is shown in Fig. 1.

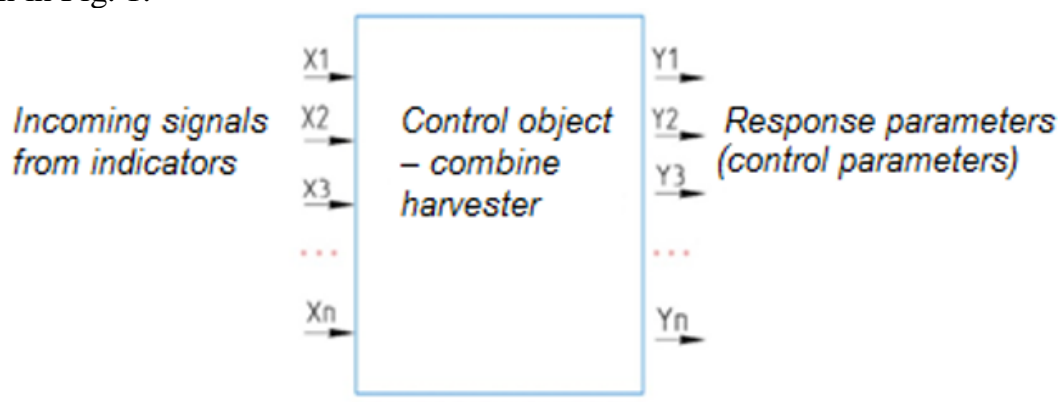

$\mathrm{X}_{1}$ - humidity of the straw heap; $\mathrm{X}_{2}-$ mass of straw heap; $\mathrm{X}_{3}$ - physical and mechanical properties of straw and grain; $X_{n}$ - complex indicator of incoming signals; $Y_{1}$ - grain trauma; $Y_{2}-$ grain loss; $\mathrm{Y}_{3}$ - completeness of grain cleaning; $\mathrm{Y}_{\mathrm{n}}$ - comprehensive indicator of the quality of the combine harvester

Fig.1. Diagram of incoming signals and output parameters.

According to the degree of autonomy of MRC and the development of their control systems, several options for the concept will be considered:

1) fully autonomous to carry out the entire list of technological operations performed in precision farming and their interaction with the service infrastructure;

2) partial autonomy with control using an operations center by means of global positioning and navigation devices with local stations (portable base stations), as well as the use of machine vision;

3) control using the operations center or control panel using global and local positioning and navigation devices with elements of partial automation.

When comparing various concepts for developing control systems of unmanned mobile energy devices (MED), the following quality criteria were used:

- directional stability of the object - MED;

- accuracy of terrain positioning with the combined use of various technical devices (global positioning systems, local positioning systems, machine vision systems, electronic field maps);

- a list of technological units of a complete MED, transferred to automatic control mode;

- accuracy of technological operations through unmanned MED;

- manufacturing costs and operating costs of an unmanned control system.

An integral indicator in assessing the various options under consideration is:

$$
Y=\sum_{k=1}^{n} a_{i} y_{i}
$$

where $a_{i}$-weight coefficient of the i-th quality criterion, $\mathrm{y}_{\mathrm{i}}$ - relative value of the $\mathrm{i}$-th quality criterion, $\mathrm{n}-$ number of criteria.

Estimation of directional stability of MRC, as a quality criterion, is carried out according to the standard deviation from the course of movement:

$$
\sigma=\sqrt{\sum_{i=1}^{N} \frac{\left(\dot{X}-X_{i}\right)^{2}}{N}}
$$

where $\mathrm{N}$ - number of quantization points of the trajectory with a step $\Delta t, \mathrm{X}_{\mathrm{i}}-$ current value of the deviation, $X$ - mathematical expectation of the deviation. 
Due to the lack of expert estimates of the weight coefficient of the quality criteria of the control parameters $a_{i}$, all the coefficients are taken equal to 1 .

According to the results of the analysis, it can be said that the most effective, based on the set of quality criteria for the control system of an unmanned combine harvester, is the concept of combined positioning devices used on MRC: the GPS/GLONASS satellite system, the base station for signal correction and machine vision using optical cameras and neural computers.

As local means, we mean local positioning and navigation systems - technologies with a sufficiently large coverage area, which provides MRC comparable to global navigation satellite system (GNSS) accuracy of coordinate-time determinations together with portable base stations.

When choosing the most effective version of the MRC control system (CS), various MS options were considered, and their comparison was carried out according to the above quality criteria. All considered options can be divided into 3 directions.

In the first direction of the concept, the following features of the applied elements for its implementation can be distinguished.

As a vision system in the developed MRC, an algorithm is used to identify various obstacles that are dangerous during the operation of agricultural machinery. To accomplish this task, video cameras and intelligent systems are used. Such a system determines the parameters of the field and recognizes obstacles.

The combine harvester control system in manual mode is a complex process: the operator must perform several functions simultaneously:

- monitor the operation of the cutting apparatus and reel of the header;

- maintain a certain speed and directional stability of the combine harvester;

- adjust the gaps of the thresher;

- control the level of loading the tank with grain;

- monitor the position of the equipment.

All these operations can be undertaken by an artificial intelligence adapted to the harvester.

Thus, the most optimal solution is the development of harvesting technology, the implementation of which eliminates or reduces damage to grain. With increased trauma to the seeds of grain crops on the working bodies of the harvesting machine, it is necessary to regulate the operating and technological parameters of the harvesting machine elements depending on the changing harvesting conditions.

Based on the foregoing, it follows that in order to increase productivity while improving the quality of grain harvesting, it is necessary to create a system of functioning elements of the harvesting machine with the determination of the magnitude of the changing external exposure-X, soil-climatic conditions in order to adapt the functions of the state- $Z$, and the control action- $U$, changing the operating and technological parameters of the working bodies of the combine harvester when ingathering grain crops to changing environmental conditions with the aim to ensure the optimal resulting indicator-Y of the harvester operating when ingathering crops.

By the factor causing the deviation of the control quantity (in this case, the resulting indicator $\mathrm{Y}$ of the harvester operating for ingathering crops) is understood the disturbing effect (external influence $\mathrm{X}$ of the soil-climatic conditions), it corresponds to the principle of disturbance control. When control by disturbance, the task is to compensate for the influence of the disturbing effect on the control quantity.

In the second direction of the concept, the following features of the applied elements can be distinguished.

In this case, a kinematic global real-time positioning system and a GPS-compass are used as navigation sensors. Sensors provide the projected prototype MRC with information 
about the position of the object and indicate the direction necessary for movement to the intended target. A mathematical tool such as a Kalman filter [2] is used for control, which estimates the errors of directional stability and lateral movements of the wheels contained in the data on the position and speed of movement. During the experimental studies, it is assumed that MRC will be able to automatically follow the target course and harvest.

Existing positioning systems are based on the use of the following technologies: satellite tracking, positioning in mobile networks, radio frequency identification, combinations of several technologies. The local base station can be controlled automatically and remotely from a personal computer and developed specialized software. It can also work autonomously, without operator intervention. It can generate satellite data for subsequent processing with data from field satellite measurements, as well as differential corrections to determine the exact spatial coordinates in real time. Data for postprocessing can be archived to the hard drive of the base station computer and transmitted via communication channels to the server for remote access. Mobile receiver users can obtain differential corrections over the air, high-speed wireless networks, or over the Internet.

To solve the problem of analysis, or positioning, a certain set of technical means is used. The composition of the tools used includes a programmable logic controller, a bot computer, as well as a set of sensors necessary for the implementation of autonomous work. Sensors provide information on the environment; based on the data received, the control system control the MRC actuators.

There are ready-made modules that solve positioning problems, but, as a rule, they are directed to a strictly defined type of conditions of use and the MRC itself [3].

The most noteworthy are the operation algorithms that are used in ready-made technical solutions, however, it should be noted that in most cases they are closed to the public. Note that the software also has an individual character, applicable to certain conditions. Thus, there are no ready-made, universal solutions for all navigation problems and often, navigation systems are developed individually for the designed MRC or environmental conditions. When evaluating the navigation methods used in MRC, the accuracy of positioning should be considered first. It is generally accepted that the permissible error in determining the coordinates of MRC is a value whose magnitude is not more than half of the minimum dimensions of the investigated object. The environment introduces fundamental inaccuracy and uncertainty into communication channels. In such circumstances, it is worth considering the ability of systems to work in a noisy environment [4].

Depending on the classification, several types of navigation systems are distinguished. Four types of movement are offered for moving along a given trajectory: global (GPS, Glonass use satellites for positioning, determine absolute coordinates), local (some point for positioning is used, usually a start point, systems using rangefinders are often used: laser, infrared, ultrasound), personal (used when positioning individual parts of MRC within a specific territory) and autonomous (gyroscopes, digital compasses are used when transmitting or receiving signals difficult or impossible from outside) of the system.

The most popular approach to solving the navigation problem is to load a local map of the terrain into the memory of the MRC on-board computer and supply it with a satellite positioning system [5].

But with such a solution to the navigation problem, the system is tightly connected with the satellite operation, and in the event of GPS or GLONASS blocking, the MRC will practically lose its orientation in space. Therefore, the on-board navigation module should also be able to build a route and control the motion parameters based on data received from the on-board sensors. Modern navigation systems are developed on the basis of interconnected modules, combining low-level control mechanisms and general route 
planning. There are two main approaches to building a navigation system: the use of centralized or distributed control architecture. With centralized control, all the calculations necessary to generate the input robot motion control vector are performed on one computer. Depending on the task, computer resources for centralized control may be too costly. In a distributed control system, instead of one main controller, individual controllers are used to control individual actuators. Distributed control can be implemented on the basis of a single-board computer or on a distributed computing platform. The control controller coordinates the operation of the individual controllers connected to it. Problems directly related to movement on the current short segment of the route are solved by the control and coordination module, and the global system monitors the observance of the general plan and, in case of deviation, corrects the route of movement and synchronizes the work of all subordinate control structures [6].

Currently, the use of the distributed control system of MRC can dramatically reduce the cost of the navigation complex and the entire system as a whole, and also makes it possible to create an autonomous on-board navigation system that solves the problem of movement in real time.

In the third direction of the concept, the following features of the applied elements for its implementation can be distinguished.

Technological processes in the considered option are carried out with the direct participation of a human -operator, and some include the automatic execution of individual production operations (the use of drives to control individual components of the combine harvester).

The simplest form of control is manual. The development of command information and determination of the impact on the object are based on the correct perception by the senses and the experience of the operator. For remote control of the technological process, in addition to receiving telemetric information, it is necessary to transmit video images of the work in real time.

With the distance between the MRC and the operator performing control within the line of sight, this problem is solved at $2.4 \mathrm{GHz}$ radio frequencies. Of these standards, Wi-Fi has the largest data transmission range of up to $1000 \mathrm{~m}$ and a maximum speed of 150-600 $\mathrm{Mbit} / \mathrm{s}$. If it is necessary to provide control of the unit out of direct visibility, then there is a group of radio frequency ranges $434 / 868 \mathrm{MHz}$ open for free use around the world, in which there are no wireless standards. At these frequencies, it is possible to provide a communication range of up to $10 \mathrm{~km}$ [7]. The positioning accuracy of the GLONASS satellite system is $10 \div 15 \mathrm{~cm}[8,9]$.

At present, precision farming technology is being introduced into agricultural production, and this requires positioning accuracy of $3 \div 5 \mathrm{~cm}$. Therefore, the idea of joint use of GLONASS/GPS is proposed, in connection with which the term appeared - global navigation satellite system (GNSS). To reduce the errors in determining the coordinates due to atmospheric and multipath effects, both instrumental and computational methods are used. As the main instrumental method, which allows increasing the accuracy of coordinate calculations, the differential GNSS operation mode is used $[10,11,12]$.

In differential mode, base stations (BS) are used on which high-precision GNSS receivers are installed. When a satellite signal is received, corrections between the calculated instantaneous coordinate values and the known BS coordinates are evaluated. These corrections allow to increase the accuracy of object positioning up to $2 \div 5 \mathrm{~cm}$.

When using computational methods, complex mathematical models are used to determine pseudo ranges and coordinates.

Based on the considered areas of the concept for creating the MRC control system, the second direction of the construction concept should be considered the most suitable development direction and created an experimental model of the MRC control system on its 
basis. It is predicted that the introduction of electrical and electronic components in the control system will significantly increase operational performance, and due to the high degree of automation of technological processes, the productivity of the combine harvester as a whole will increase. The experimental model of MRC is created universal and with the help of components and assemblies can harvest selective and seed crops, as well as harvest grain, leguminous and rice in farms with a sowing area of at least 50 hectares. The undercarriage system of the experimental MRC model will be equipped with sensors and an unmanned control system.

The implementation of the MRC control system according to the chosen concept of creation is necessary for the subsequent integration of the obtained project solutions into the federal and regional digital platforms of the agro-industrial complex based on the platform model of the PAAS-model for providing cloud computing, in which the consumer gains access to the use of information platforms: operating systems, database control systems.

The key result of this development is to increase the productivity of agricultural machinery by 2-3 times, to improve the quality of harvesting, and also to improve working conditions when harvesting through the use of unmanned control system of MRC.

\section{Conclusions}

1. When choosing the most suitable direction of the concept for creating an MRC control system, one should adhere to the second concept of building an MRC.

2. Increased autonomy of MRC is ensured through control from the operations center using global positioning and navigation means (GPS/GLONASS), together with local stations (portable base stations), machine vision and various external signal sensors.

3. The MRC control system is an automated complex system based on artificial intelligence, which will include mechanical, electrical and electronic components (a video camera, a neural network computer with additional units for interfacing with the on-board systems of the combine harvester, controllers, etc.).

4. The concept of creating the MRC control system establishes the need for subsequent integration of the obtained project decisions into the federal and regional digital platforms of the agro-industrial complex of Russia on the basis of the PAAS cloud platform model. Its implementation will increase the productivity of the combine harvester by 2-3 times.

This paper was completed as part of a grant agreement with the Ministry of Science and Higher Education.

\section{References}

1. A.A. Boguslavsky, G.K. Borovin, V.A. Kartashev, V.E. Pavlovsky, S.M. Sokolov, Models and algorithms for intelligent control systems, (2019). doi: 10.20948/mono2019-boguslav

2. E.V. Zhalnin, Z.A. Godzhaev, S.N. Florentsev, Agricultural Machines and Technologies, 6, 9-16, (2017). doi: 10.22314/2073-7599-2017-6-9-16

3. Z.A. Godzhaev, A.P. Grishin, A.A. Grishin, Tractors and agricultural machinery 12, 42-45 (2015). doi: 10.22314/2073-7599-2018-12-1-37-41

4. N.T. Goncharov, A.Yu. Izmailov, V.K. Khoroshenkov, I.G. Smirnov, I.I. Afonina, E.S. Luzhnova, I.S. Alekseev, S.E. Lonin, Automation in industry 1, 38-40, (2017). doi:10.22314/2073-7599-2018-12-6-48-52 
5. A.Yu. Izmailov, V.K. Khoroshenkov, V.A. Kolesnikova, I.S. Alekseev, S.E. Lonin, N.T. Goncharov, Agricultural machinery and technology 3, 3-9, (2017), doi:10.22314/2073-7599-2017-3-3-9

6. Yu.F. Lachuga, A.Yu. Izmailov, Ya.P. Lobachevsky, N.K. Mazitov, Agricultural machinery and technology 2, 37-42, (2017). doi: 10.22314/2073-7599-2017-2-37-41

7. A.Yu. Izmailov, Y.P. Lobachevsky, Yu.S. Tsench, E.S. Luzhnova, I.S. Alekseev, E.N. Ilchenko, S.E. Lonin, Bulletin of the Russian Agricultural Science 4, 63-68, (2019). doi: 10.30850/vrsn/2019/4/63-68

8. O. C. Barawid, A. Mizushima, K. Ishii, N. Noguchi, Biosystems Engineering 96(2), 139-149, (2007). doi:10.1016/j.biosystemseng.2006.10.01

9. M. Iida, Y. Yamada. In Proceedings of the Conference of Automation Technology for Off-road Equipment, 125-131, (2006). doi: 10.1109/ICMA.2011.5985704

10. Mahalakshmi, J., Kuppusamy, K., Kaleeswari, C., \& Maheswari, P. (2019). Emerging Technologies for Agriculture and Environment, 39-52. doi:10.1007/978-981-137968-04

11. Q. Chen, C. Liao, A. Ouyang, X. Li, Q. Xiao, International Journal of Electric and Hybrid Vehicles, 8(3), 242, (2016). doi:10.1504/ijehv.2016.08002

12. Y.P. Lobachevsky, S.E Lonin, I.S. Alekseev, N.T. Goncharov, I.I. Afonina, E.N. Ilchenko, Agricultural machinery and technology 13(2), 48-52, (2019) doi:10.22314/2073-7599-2018-12-6-48-52 